SOME PROPERTIES OF TUBULAR MINIMAL SURFACES OF ARBITRARY CODIMENSION

This content has been downloaded from IOPscience. Please scroll down to see the full text. 1991 Math. USSR Sb. 68133

(http://iopscience.iop.org/0025-5734/68/1/A07)

View the table of contents for this issue, or go to the journal homepage for more

Download details:

IP Address: 130.236.73.7

This content was downloaded on 10/11/2014 at 11:29

Please note that terms and conditions apply. 


\title{
SOME PROPERTIES OF TUBULAR MINIMAL SURFACES OF ARBITRARY CODIMENSION
}

\author{
V. M. MIKLYUKOV AND V. G. TKACHEV
}

\begin{abstract}
A tubular surface is an immersion $u: M \rightarrow \mathbf{R}^{n}$ for which the section $\Pi \cap u(M)$ by an arbitrary hyperplane $\Pi$ orthogonal to a fixed vector $e \in \mathbf{R}^{n}$ is a compact set.

For tubular minimal surfaces in $\mathbf{R}^{n}$ we prove that

(a) if $\operatorname{dim} M=2$ and $u(M)$ lies in a half-space, then $u(M)$ also lies in some hyperplane; and

(b) if $\operatorname{dim} M \geq 3$, then a tubular minimal surface lies in the layer between two hyperplanes orthogonal to $e$.

We obtain the corresponding results about the structure of the Gaussian image of two-dimensional tubular minimal surfaces.

The case $\operatorname{codim} M=1$ was investigated earlier (Math. USSR Sb. 59 (1988), 237-245 (MR 88e:53009)).

Bibliography: 19 titles.
\end{abstract}

Let $M$ be a $p$-dimensional connected oriented noncompact manifold of class $C^{2}$. Consider the surface $(M, u)$ given by a $C^{2}$-immersion $u: M \rightarrow \mathbf{R}^{n}$, where $2 \leq p \leq n-1$. Everywhere below we assume that the immersion $u$ is proper; that is, the inverse image of any compact set $F \subset \mathbf{R}^{n}$ is a compact set in $M$.

The surface $(M, u)$ is said to be minimal if its mean curvature vector $H$ vanishes identically ([1], p. 34).

Let $M$ be a manifold without boundary. We shall say that the surface $(M, u)$ is tubular if there exist a vector $e_{0} \in \mathbf{R}^{n}$ and two numbers $-\infty \leq a<b \leq+\infty$ such that for any hyperplane $\Pi_{t}=\left\{x \in \mathbf{R}^{n}:\left\langle x, e_{0}\right\rangle=t\right\}$ orthogonal to $e_{0}$ the section $\Sigma_{e_{0}}(t)=u(M) \cap \Pi_{t}$ is not empty for any $t \in(a, b)$ and any portion included between two hyperplanes $\Pi_{t_{1}}$ and $\Pi_{t_{2}}$ with $a<t_{1}<t_{2}<b$ is a compactum. In this case the interval $(a, b)$ will be called the projection of the surface $(M, u)$, and the finite or infinite difference $b-a$ will be called its length.

We shall say that the surface $(M, u)$ is tubular in the large if it is tubular with projection $(-\infty,+\infty)$.

The simplest example of a minimal surface in $\mathbf{R}^{3}$ that is tubular in the large is a catenoid. Numerous examples of two-dimensional immersed tubular minimal surfaces in $\mathbf{R}^{n}$ can easily be constructed by starting from the representation for such surfaces given in [2].

1980 Mathematics Subject Classification (1985 Revision). Primary 53A10; Secondary 53A07. 
In the theorems formulated below we shall assume that $\operatorname{codim} M>1$. The case of tubular minimal surfaces of codimension 1 is special and is considered in [3]. Tubular minimal surfaces with nontrivial symmetry groups are studied in the series of papers [14]-[16].

THeOREM 1. Let $(M, u)$ be a two-dimensional minimal surface in $\mathbf{R}^{n}$ that is tubular in the large. If there is a hyperplane $\Pi$ that does not intersect $u(M)$, then the set $u(M)$ lies in some hyperplane $\Pi_{1}$ parallel to $\Pi$.

This theorem was announced in [4].

The property of a two-dimensional minimal surface of being tubular in the large is specific. Namely, as the following assertion shows, any tubular minimal surface of dimension greater than 2 has finite length.

THEOREM 2. Let $(M, u)$ be a tubular minimal surface in $\mathbf{R}^{n}$ of dimension $p \geq 3$. Then the set $u(M)$ is situated in the layer between two parallel hyperplanes orthogonal to the vector $e_{0}$.

In [3] for $\operatorname{codim} M=1$ an exact estimate was given for the length of a tubular minimal surface. It is not known whether a similar estimate is possible in the case of arbitrary codimension.

Let $G_{n}^{2}$ be the Grassmann manifold of oriented two-dimensional planes passing through the origin, with canonical distance $\theta\left(\gamma_{1}, \gamma_{2}\right)$ (that is, the angle between the planes $\gamma_{1}, \gamma_{2} \in G_{n}^{2}$ ). In the case of planes $\gamma_{1}$ and $\gamma_{2}$ oriented in the same (respectively, opposite) way, $\theta\left(\gamma_{1}, \gamma_{2}\right)$ is defined as the maximum angle formed by all possible pairs of lines from $\gamma_{1}$ and $\gamma_{2}$ (respectively, the complement of this maximum angle with respect to $\pi$ ).

Let $m \in M$ be an arbitrary point. Then the tangent plane $T_{m}(M)$ at the point $u(m)$ to the surface $(M, u)$ is parallel to some two-dimensional plane $m^{*} \subset G_{n}^{2}$ with the same orientation as $T_{m}(M)$. The correspondence $m \rightarrow m^{*}$ is called the Gaussian map of the surface $(M, u)$. Let $M^{*}$ denote the image of $M$ under this map, and put

$$
\delta\left(M^{*}\right)=\inf _{\gamma_{1} \in G_{n}^{2}} \sup _{\gamma_{2} \in M^{*}} \theta\left(\gamma_{1}, \gamma_{2}\right)
$$

Clearly, $\delta\left(M^{*}\right) \leq \pi$ by the definition of the angle $\theta\left(\gamma_{1}, \gamma_{2}\right)$.

It is convenient to introduce the following definitions, which make the geometrical nature of the assertion formulated below more prominent. For an arbitrary plane $\gamma \in$ $G_{n}^{2}$ we consider the equator $K_{\gamma}$, that is, the set $K_{\gamma}=\left\{\xi \in G_{n}^{2}: \theta(\xi ; \gamma)=\pi / 2\right\}$, which is a hypersurface in $G_{n}^{2}$, and the analogous equator of the Euclidean sphere. The set $G_{n}^{2} \backslash K_{\gamma}$ splits into two connected components, called the halves of the Grassmannian $G_{n}^{2}$. Clearly, the planes $\xi \in G_{n}^{2}$ and $\xi^{-}$with the opposite orientation to $\xi$ always lie in different halves of $G_{n}^{2}$.

The following assertion says that the closure of the Gaussian image of a minimal surface that is tubular in the large cannot lie in half of $G_{n}^{2}$.

THEOREM 3. Let $(M, u)$ be a two-dimensional minimal surface in $\mathbf{R}^{n}$ that is tubular in the large. Then $M^{*}$ intersects any equator of $G_{n}^{2}$, or in other words $\delta\left(M^{*}\right) \geq \pi / 2$. 
We observe that the arguments we use to prove these theorems can be applied to study the structure of the Gaussian image of minimal surfaces that are tubular in the large close to infinity in terms of the ends. In this direction there are qualitatively different approaches that rely on the asymptotic growth of the scalar curvature and the topological type of the manifold $M$ in [18] and [19], for example.

All the results we have mentioned have a function-theoretic nature. From what follows it will be clear that they are none other than the geometrical interpretations of theorems of Liouville type on manifolds for harmonic (superharmonic) functions. At the base of the arguments lies the concept of the capacity of a condenser on a surface [5], [6].

\section{$\S 2$}

Let us agree on notation. Let $(M, u)$ be a surface given by an immersion $u: M \rightarrow$ $\mathbf{R}^{n}$. The standard metric on $\mathbf{R}^{n}$ induces a Riemannian metric on $M$ under the map $u$. Henceforth $M$ will be regarded as a Riemannian manifold endowed with this metric.

Let $T(M)$ and $N(M)$ denote the tangent and normal bundles on $M$, and let $T_{m}=T_{m}(M)$ and $N_{m}=N_{m}(M)$ denote the tangent and normal spaces at a point $m \in M$. For a pair of vectors $X$ and $Y$ in $T_{m}$ the symbol $\langle X, Y\rangle$ will denote their inner product. Let $\bar{\nabla}$ and $\nabla$ denote connections in $\mathbf{R}^{n}$ and $T(M)$ (or $N(M)$ ) respectively, under which for any sections $X$ and $Y$ of the bundle $T(M)$ and section $\xi$ of the bundle $N(M)$ we have

$$
\nabla_{X} Y=\left(\bar{\nabla}_{X} Y\right)^{T}, \quad \nabla_{X} \xi=\left(\bar{\nabla}_{X} \xi\right)^{N},
$$

where we agree that the symbol $(v)^{T}$ (or $(v)^{N}$ ) denotes the orthogonal projection of a vector $v \in \mathbf{R}^{n}$ on the tangent (normal) space at the corresponding point.

The second quadratic form $B$ is defined as the bilinear symmetric map from $T_{m} \otimes T_{m}$ to $N_{m}$ dual to the Weingarten homomorphism $A^{\xi}$ of the tangent space onto itself. We have

$$
A^{\xi} X=-\left(\bar{\nabla}_{X} \xi\right)^{T}, \quad\langle B(X ; Y) ; \xi\rangle=\left\langle A^{\xi}(X) ; Y\right\rangle
$$

for any $\xi \in N_{m}$ and $X, Y \in T_{m}$ (see [7], for example).

We shall need the following auxiliary assertion.

LEMMA 1. Let $E_{1}, \ldots, E_{p}$ be an orthonormal basis of $T_{m}(M)$ and let $\xi$ and $Y$ be arbitrary sections of $N(M)$ and $T(M)$ respectively. Then

$$
\operatorname{div}\left(A^{\xi} Y\right)=\sum_{i=1}^{p}\left(\left\langle B\left(E_{i}, Y\right) ; \nabla_{E_{i}} \xi\right\rangle+\left\langle A^{\xi} E_{i} ; \nabla_{E_{i}} Y\right\rangle\right)+\left\langle\nabla_{Y} H, \xi\right\rangle,
$$

where $H$ denotes the mean curvature vector of the surface $(M, u)$.

Proof. We first mention the identity

$$
\nabla_{E} A^{\xi}(Y)=\nabla_{Y} A^{\xi}(E)+A^{\nabla_{E} \xi}(Y)-A^{\nabla_{Y} \xi}(E)+A^{\xi}([E, Y]),
$$

where $[E, Y]=\nabla_{E} Y-\nabla_{Y} E$ is the commutator of the vector fields $E$ and $Y$.

The truth of (3) follows from the chain of equalities

$$
\begin{aligned}
\nabla_{E} A^{\xi}(Y) & =\left(\bar{\nabla}^{\xi}(Y)\right)^{T}=-\left(\bar{\nabla}_{E}\left(\bar{\nabla}_{Y} \xi\right)^{T}\right)^{T}=-\left(\bar{\nabla}_{E} \bar{\nabla}_{Y} \xi\right)^{T}+\left(\bar{\nabla}_{E} \nabla_{Y} \xi\right)^{T} \\
& =-\left(\bar{\nabla}_{Y} \bar{\nabla}_{E} \xi+\bar{\nabla}_{[E, Y]} \xi\right)^{T}-A^{\nabla_{Y} \xi}(E) \\
& =-\left(\bar{\nabla}_{Y}\left(-A^{\xi}(E)\right)+\bar{\nabla}_{Y} \nabla_{E} \xi\right)^{T}+A^{\xi}([E, Y])-A^{\nabla_{Y} \xi}(E) .
\end{aligned}
$$


Let us use (3). We have

$$
\begin{aligned}
\operatorname{div} A^{\xi}(Y)= & \sum_{i=1}^{p}\left\langle E_{i}, \nabla_{E_{i}} A^{\xi} Y\right\rangle=\sum_{i=1}^{p}\left\langle E_{i}, \nabla_{Y} A^{\xi} E_{i}\right\rangle+\sum_{i=1}^{p}\left\langle E_{i}, A^{\nabla_{E_{i}}}(Y)\right\rangle \\
& -\sum_{i=1}^{p}\left\langle E_{i} A^{\nabla_{Y} \xi}\left(E_{i}\right)\right\rangle+\sum_{i=1}^{p}\left\langle E_{i}, A^{\xi}\left(\left[E_{i}, Y\right]\right)\right\rangle=\Sigma_{1}+\Sigma_{2}-\Sigma_{3}+\Sigma_{4} .
\end{aligned}
$$

Let us find $\Sigma_{2}$ and $\Sigma_{3}$ :

$$
\begin{gathered}
\Sigma_{2}=\sum_{i=1}^{p}\left\langle B\left(E_{i}, Y\right), \nabla_{E_{i}} \xi\right\rangle \\
\Sigma_{3}=\sum_{i=1}^{p}\left\langle B\left(E_{i}, E_{i}\right), \nabla_{Y} \xi\right\rangle=\left\langle\operatorname{trace} B, \nabla_{Y} \xi\right\rangle=\left\langle H, \nabla_{Y} \xi\right\rangle .
\end{gathered}
$$

Let $A$ be a section of the bundle whose fiber at each point $m \in M$ coincides with the space of symmetric homomorphisms $T_{m}(M) \rightarrow T_{m}(M)$. Then for any orthonormal basis $E_{1}, \ldots, E_{p}$ of the space $T_{m}(M)$ and any vector $Y \in T_{m}(M)$ we have

$$
\sum_{i=1}^{p}\left\langle A E_{i}, \nabla_{Y} E_{i}\right\rangle=0 .
$$

For it is sufficient to observe that the sum in (5) does not depend on the choice of basis $E_{1}, \ldots, E_{p}$, since in the special basis consisting of proper fields of the section $A$ (its local existence is a consequence of the symmetry of $A$ ) this sum takes the form

$$
\sum_{i=1}^{p}\left\langle A E_{i}, \nabla_{Y} E_{i}\right\rangle=\sum_{i=1}^{p} \lambda_{i}\left\langle E_{i}, \nabla_{Y} E_{i}\right\rangle=0
$$

We now consider an arbitrary basis $F_{1}, \ldots, F_{p}$. Let

$$
F_{j}=\sum_{i=1}^{p} \alpha_{j i} E_{i}, \quad \sum_{i=1}^{p} \alpha_{j i} \alpha_{j k}=\delta_{j k},
$$

where $\delta_{j k}$ is the Kronecker symbol. Then

$$
\begin{aligned}
\sum_{j=1}^{p}\left\langle A F_{j}, \nabla_{Y} F_{j}\right\rangle & =\sum_{j=1}^{p} \sum_{i, k=1}^{p}\left\langle A E_{i}, E_{k} \nabla_{Y} \alpha_{j k}+\alpha_{j k} \nabla_{Y} E_{k}\right\rangle \alpha_{j i} \\
& =\sum_{i=1}^{p}\left\langle A E_{i}, \nabla_{Y} E_{i}\right\rangle+\sum_{i, k=1}^{p}\left(\left\langle A E_{i}, E_{k}\right\rangle \sum_{j=1}^{p} \alpha_{j i} \nabla_{Y} \alpha_{j k}\right)
\end{aligned}
$$

Since $\left\langle A E_{i}, E_{k}\right\rangle$ is symmetric and $\sum_{j=1}^{p} \alpha_{j i} \nabla_{Y} \alpha_{j k}$ is skew-symmetric in the indices $i$ and $k$, the last term vanishes.

Using (5), it is easy to calculate $\Sigma_{1}$ and $\Sigma_{4}$ :

$$
\begin{aligned}
\Sigma_{1}=\sum_{i=1}^{p}\left\langle E_{i}, \nabla_{Y}\left(A^{\xi} E_{i}\right)\right\rangle & =-\sum_{i=1}^{p}\left\langle A^{\xi} E_{i}, \nabla_{Y} E_{i}\right\rangle+\sum_{i=1}^{p} \nabla_{Y}\left\langle A^{\xi} E_{i}, E_{i}\right\rangle \\
& =\nabla_{Y} \operatorname{trace} A^{\xi}=\nabla_{Y}\langle H, \xi\rangle ;
\end{aligned}
$$




$$
\Sigma_{4}=\sum_{i=1}^{p}\left\langle E_{i}, A^{\xi}\left[E_{i}, Y\right]\right\rangle=\sum_{i=1}^{p}\left\langle A^{\xi} E_{i}, \nabla_{E_{i}} Y-\nabla_{Y} E_{i}\right\rangle=\sum_{i=1}^{p}\left\langle A^{\xi} E_{i}, \nabla_{Y} E_{i}\right\rangle .
$$

Substituting the expressions we have found for the sums $\Sigma_{i}$ in (4), we arrive at the required relation (2).

\section{$\S 3$}

Let $M$ be a Riemannian manifold with boundary $\partial M$ (possibly empty). Let $P, Q \subset M$ be disjoint closed sets. We define the capacity of the condenser $(P, Q)$ by putting

$$
\operatorname{cap}(P, Q)=\inf \int_{M}|\nabla \varphi|^{2},
$$

where $|\nabla \varphi|^{2}=\langle\nabla \varphi, \nabla \varphi\rangle$ is the scalar square of the gradient $\nabla \varphi$ and the infimum is taken over all locally Lipschitz functions $\varphi: M \rightarrow \mathbf{R}^{1}$, where $\varphi(M)=1$ when $m \in P$ and $\varphi(m)=0$ when $m \in Q$.

We shall say that a manifold has parabolic type if for any compactum $F \subset M$ there is an exhaustion $D_{1} \subset D_{2} \subset \cdots, \bigcup_{1}^{\infty} D_{k}=M$ of $M$ by a sequence of open sets $D_{k} \supset F$ with compact closures, for which

$$
\lim _{k \rightarrow \infty} \operatorname{cap}\left(F, M \backslash D_{k}\right)=0 .
$$

The assertion made below about the type being parabolic is a key assertion in the constructions, and is of independent interest. Some other tests for the type of two-dimensional minimal surfaces to be parabolic can be found in [8] and [9].

THEOREM 4. Any minimal surface $(M, u)$ in $\mathbf{R}^{n}$ that is tubular in the large has parabolic type.

It is advisable to carry out part of the proof in a somewhat more general form than is required by the conditions. We first give the necessary definition.

Consider a manifold $M$ with boundary $\partial M \neq \varnothing$, and call a surface $(M, u)$ a band with projection $(a, b)$ if there is a vector $e_{0} \in \mathbf{R}^{n}$ such that the following three conditions hold:

(i) Any section $u(M) \cap \Pi_{t}=\Sigma_{e_{0}}(t)$ is not empty when $t \in(a, b)$ and contains a point of $u(\partial M)$.

(ii) Any portion $u(M)$ included between two hyperplanes $\Pi_{t_{1}}$ and $\Pi_{t_{2}}$, where $a<t_{1}<t_{2}<b$, is a compact set in $M$.

(iii) Everywhere on $\partial M$ we have $\left\langle\nu, e_{0}\right\rangle=0$, where $\nu$ is the inward normal on $M$ to the boundary $\partial M$, or equivalently $\nabla_{\nu} f=0$ everywhere on $\partial M$ for $f=\left\langle u(m), e_{0}\right\rangle$.

A simple example of a two-dimensional minimal band with projection $(-\infty,+\infty)$ (as in the case of tubular surfaces, such bands will be called bands in the large) is the part of the helicoid cut out by a coaxial cylinder of finite radius. It is also easy to verify that the set $\left\{x=\left(x_{0}, x_{1}, \ldots, x_{p}\right) \in \mathbf{R}^{p+1}: \sum_{i=1}^{p-1} x_{i}^{2} \leq 1, x_{p}=0\right\}$ is a flat minimal band in the large of codimension 1 for any $p \geq 2$. Thus, in contrast to tubular minimal surfaces, minimal bands in the large exist for all dimensions.

We have borrowed the idea of considering minimal bands from the theory of a relativistic string (see [10] and [11], for example), in which minimal bands and tubular surfaces (but in Minkowski space-time $\mathbf{R}_{1}^{n}$ ) are the most important object of investigation. The study of a similar object in $\mathbf{R}^{n}$ also seems promising. 
For any $t \in(a, b)$ we define the sets

$$
P(t)=\{m \in M: f(m) \leq t\}, \quad Q(t)=\{m \in M: f(m) \geq t\},
$$

where as before $f(m)$ denotes the coordinate function $f(m)=\left\langle u(m), e_{0}\right\rangle$.

We also put

$$
M\left(t_{1}, t_{2}\right)=M \backslash\left(P\left(t_{1}\right) \cup Q\left(t_{2}\right)\right) .
$$

LEMMA 2. Let $(M, u)$ be a tubular minimal surface (or band) in $\mathbf{R}^{n}$ with projection $(a, b)$. Then for any $\left(t_{1}, t_{2}\right)$ from the interval $(a, b)$ we have

$$
\operatorname{cap}\left(P\left(t_{1}\right), Q\left(t_{2}\right)\right)=I /\left|t_{2}-t_{1}\right|,
$$

where $I$ is a constant independent of $t_{1}$ and $t_{2}$.

Proof. Without loss of generality we may assume that $t_{1}$ and $t_{2}$ are regular values of the function $f(m)$. We define a function $\varphi(m)=\varphi$ by

$$
\varphi(m)=\frac{t_{2}-f(m)}{t_{2}-t_{1}}
$$

where $m \in M\left(t_{1}, t_{2}\right)$, defining it as 1 when $m \in P\left(t_{1}\right)$ and 0 when $m \in Q\left(t_{2}\right)$. This function is admissible in the variational problem (6) for the condenser $\left(P\left(t_{1}\right), Q\left(t_{2}\right)\right)$, and so

$$
\operatorname{cap}\left(P\left(t_{1}\right), Q\left(t_{2}\right)\right) \leq \int_{M}|\nabla \varphi|^{2}=\frac{1}{\left(t_{2}-t_{1}\right)^{2}} \int_{M\left(t_{1}, t_{2}\right)}|\nabla f|^{2} .
$$

We also observe that $f(m)$ is a linear combination of harmonic functions on $M$ since the surface $(M, u)$ is minimal (see [1], p. 340), and hence $\Delta f=0$ everywhere on $M$.

Since the immersion $u$ is proper, the sections $\Sigma_{e_{0}}(t)$ are compact and for regular $t$ they are submanifolds of $M$. Using Stokes's formula, we obtain

$$
\begin{aligned}
& \int_{M\left(t_{1}, t_{2}\right)}|\nabla f|^{2}=\int_{\partial M\left(t_{1}, t_{2}\right)} f\langle\nabla f, \nu\rangle-\int_{M\left(t_{1}, t_{2}\right)} f \Delta f \\
& \quad=t_{2} \int_{\Sigma_{e_{0}}\left(t_{2}\right)}\langle\nabla f, \nu\rangle-t_{1} \int_{\Sigma_{e_{0}}\left(t_{1}\right)}\langle\nabla f, \nu\rangle+\int_{\partial M \cap M\left(t_{1}, t_{2}\right)} f\langle\nabla f, \nu\rangle .
\end{aligned}
$$

The last of the integrals in (8) vanishes by the definition of the surface $(M, u)$. Using once more the fact that $f(m)$ is harmonic, we conclude that

$$
\int_{M\left(t_{1}, t_{2}\right)} \Delta f=\int_{\Sigma_{e_{0}}\left(t_{2}\right)}\langle\nabla f, \nu\rangle-\int_{\Sigma_{e_{0}}\left(t_{1}\right)}\langle\nabla f, \nu\rangle=0 .
$$

Let $I$ denote any of the latter integrals; taking account of (7) and (8) we find that

$$
\operatorname{cap}\left(P\left(t_{1}\right), Q\left(t_{2}\right)\right) \leq \frac{1}{t_{2}-t_{1}} \int_{\Sigma_{e_{0}}(t)}\langle\nabla f, \nu\rangle=\frac{I}{t_{2}-t_{1}} .
$$

To verify that equality holds, it is sufficient to observe that $\varphi(m)$ is actually an extremal for the calculation of the capacity of the condenser $\left(P\left(t_{1}, Q\left(t_{2}\right)\right)\right.$, since it is harmonic and satisfies the "natural" boundary condition $\nabla_{\nu} f=0$ on $\partial M$ (see [12], p. 41).

Turning to the proof of Theorem 4, we specify arbitrarily a compact set $F \subset M$. We choose $t_{0}>0$ so that the set $M\left(-t_{0}, t_{0}\right)$ contains $F$. We fix $t>t_{0}$ and denote by $\varphi_{1}$ and $\varphi_{2}$ functions admissible in the variational problem (6) for the condensers $\left(P(-t), Q\left(-t_{0}\right)\right)$ and $\left(P\left(t_{0}\right), Q(t)\right)$ respectively. 
Consider the function $\left(1-\varphi_{1}\right) \varphi_{2}$. This function is equal to 1 on $M\left(-t_{0}, t_{0}\right)$ and vanishes on $M \backslash M(-t, t)$. Consequently, this function is admissible in the calculation of the capacity of the condenser $(F, M \backslash M(-t, t))$, and therefore

$$
\operatorname{cap}(F ; M \backslash M(-t, t)) \leq \int_{M\left(-t,-t_{0}\right)}\left|\nabla \varphi_{1}\right|^{2}+\int_{M\left(t_{0}, t\right)}\left|\nabla \varphi_{2}\right|^{2} .
$$
that

Since $\varphi_{1}$ and $\varphi_{2}$ are arbitrary admissible functions, from this inequality it follows

$$
\operatorname{cap}(F ; M \backslash M(-t, t)) \leq \operatorname{cap}\left(P(-t), Q\left(-t_{0}\right)\right)+\operatorname{cap}\left(P\left(t_{0}\right), Q(t)\right),
$$

and on the basis of Lemma 2

$$
\operatorname{cap}(F ; M \backslash M(-t, t)) \leq \frac{2 I}{t-t_{0}} .
$$

Making $t \rightarrow \infty$ in the last inequality, we verify the theorem.

This proof can be carried through unchanged for bands, and so we have the following result.

THEOREM $4^{\prime}$. Any minimal band in the large has parabolic type.

$\S 4$

Proof of Theorem 1. We assume that there is a hyperplane $\Pi$ in $\mathbf{R}^{n}$ for which $u(M) \cap \Pi=\varnothing$. Since minimality of the surface $(M, u)$ is invariant under the orthogonal group of transformations of space, we may assume that $u(M)$ is situated in the half-space $x_{1} \leq 0$. We show that in this case $f_{1}(m)=\left\langle u, e_{1}\right\rangle=$ const, where $e_{1} \in \mathbf{R}^{n}$ corresponds to the coordinate $x_{1}$ in $\mathbf{R}^{n}$.

Let us fix a point $m_{0} \in M$ and choose a constant $c<f_{1}\left(m_{0}\right)$. Let $O$ denote the connected component of the open set $\left\{m \in M: f_{1}(m)>c\right\}$ containing the point $m_{0}$. Clearly $O$ is not empty, and the function $f_{1}(m)-c$ is bounded on $O$ and vanishes on its boundary.

Let $F \subset O$ be an arbitrary compact set, and let $D_{1} \subset D_{2} \subset \cdots, \cup_{1}^{\infty} D_{k}=M$, be an exhaustion of $M$ by a sequence of open sets with compact closures, $D_{k} \supset F$.

We specify a locally Lipschitz function $\varphi(m)$, admissible for the calculation of the condenser $\left(F, M \backslash D_{k}\right)$. The function

$$
g(m)=\left(f_{1}(m)-c\right) \varphi^{2}(m)
$$

vanishes everywhere on the boundary of the set $O \cap D_{k}$. Using Stokes's formula, we obtain

$$
\int_{O}\left\langle\nabla g, \nabla f_{1}\right\rangle=-\int_{O} g \Delta f_{1} \text {. }
$$

Since $\Delta f_{1}(m) \equiv 0$, from the last relation it follows that

$$
\int_{O} \varphi^{2}\left|\nabla f_{1}\right|^{2}=-2 \int_{O} \varphi\left(f_{1}-c\right)\left\langle\nabla \varphi, \nabla f_{1}\right\rangle
$$

We observe that

$$
\left\langle\nabla \varphi, \nabla f_{1}\right\rangle \leq|\nabla \varphi|\left|\nabla f_{1}\right|,
$$

and apply Cauchy's integral inequality to the right-hand side of (9). We thus arrive at the inequality

$$
\int_{O} \varphi^{2}\left|\nabla f_{1}\right|^{2} \leq 2\left(\int_{O}\left(f_{1}-c\right)^{2}|\nabla \varphi|^{2}\right)^{1 / 2}\left(\int_{O} \varphi^{2}\left|\nabla f_{1}\right|^{2}\right)^{1 / 2}
$$


and therefore

$$
\int_{O} \varphi^{2}\left|\nabla f_{1}\right|^{2} \leq 4 c^{2} \int_{O}|\nabla \varphi|^{2}
$$

Taking account of the fact that $\varphi(m) \equiv 1$ when $m \in F$, and going over to the infimum over all $\varphi(m)$, we have

$$
\int_{F}\left|\nabla f_{1}\right|^{2} \leq 4 c^{2} \operatorname{cap}\left(F ; M \backslash D_{k}\right) .
$$

Let us use Theorem 4. Making $k \rightarrow \infty$, we have $\left|\nabla f_{1}\right|=0$ everywhere on $F$. In view of the arbitrary choice of $F \subset O$ we conclude that $f_{1}(m)=f_{1}\left(m_{0}\right)$ for all $m \in O$. Now taking account of the arbitrary choice of constant $c$, we see that $f_{1}(m) \equiv f_{1}\left(m_{0}\right)$ on $M$; that is, the set $u(M)$ is situated in some hyperplane.

Let us state an analog of Theorem 1 for minimal bands in the large, now not necessarily two-dimensional.

THEOREM $1^{\prime}$. Let $(M, u)$ be a minimal band in the large, situated in the halfspace $x_{1} \leq 0$. If everywhere on the boundary of the band $\partial M$ we have $\left\langle v, e_{1}\right\rangle \leq 0$ (where $e_{1} \in \mathbf{R}^{n}$ is the vector corresponding to the coordinate $x_{1}$ ), then the set $u(M)$ is situated in some hyperplane $x_{1} \equiv$ const .

The proof differs only in the details from the proof given above: namely, (9) is replaced by the inequality

$$
\int_{O} \varphi^{2}\left|\nabla f_{1}\right|^{2} \leq-2 \int_{O} \varphi\left(f_{1}-c\right)\left\langle\nabla \varphi, \nabla f_{1}\right\rangle
$$

To verify $\left(9^{\prime}\right)$ we observe that the boundary of the set $O \cap D_{k}$ can be represented as the union of the two sets $\partial\left(O \cap D_{k}\right) \cap($ int $M)$ and $\partial\left(O \cap D_{k}\right) \cap \partial M$. Everywhere on the first of these the function $\varphi^{2}\left(f_{1}-c\right)=0$, and everywhere on the second $\varphi^{2}\left(f_{1}-c\right) \geq 0$ and $\nabla_{\nu} f_{1} \leq 0$. Hence we obtain

$$
\int_{O} \varphi^{2}\left|\nabla f_{1}\right|^{2}+2 \int_{O} \varphi\left(f_{1}-c\right)\left\langle\nabla \varphi, \nabla f_{1}\right\rangle=\int_{\partial\left(O \cap D_{k}\right)} \varphi^{2}\left(f_{1}-c\right) \nabla_{\nu} f_{1} \leq 0,
$$

so $\left(9^{\prime}\right)$ is proved.

The requirement that a tubular minimal surface should lie in a half-space, imposed in Theorem 1, can be weakened somewhat. This follows from the next assertion.

THEOREM 5. Let $(M, u)$ be a two-dimensional tubular minimal surface with projection $(a,+\infty)$ in $\mathbf{R}^{n}$, and let $\mu(t)$ be the maximum value of the coordinate $x_{1}$ on the section $\Sigma_{e_{0}}(t)$. Suppose that $\mu\left(t_{0}\right) \leq \mu_{0}<\infty$ for some $t_{0} \in(a,+\infty)$. Then either $\mu(t) \leq \mu_{0}$ when $t>t_{0}$, or

$$
\lim _{t \rightarrow+\infty} \frac{\mu(t)}{\sqrt{t}}>0
$$

For the proof we suppose that $\mu(t)>\mu_{0}$ for some $t>t_{0}$. We choose a point $m_{1} \in M$ so that $f_{1}\left(m_{1}\right)>\mu_{0}$ and $f\left(m_{1}\right)=\left\langle u\left(m_{1}\right), e_{0}\right\rangle>t_{0}$. Let $O$ denote the connected component of the set $\left\{m \in M: f_{1}(m)>\mu_{0}\right\}$ containing the point $m_{1}$. We arbitrarily choose a compactum $F \subset O$.

Let $t^{\prime}=\max _{m \in F} f(m)$ and suppose that $T>t^{\prime}$. Consider the sets $P\left(t^{\prime}\right)$ and $Q(T)$. We specify a locally Lipschitz function $\varphi(m)$ that is equal to 1 on $P$ and to 
0 on $Q$. The function $g(m)=\varphi^{2}(m)\left(f_{1}(m)-\mu_{0}\right)$ has compact support contained in $O$. By Stokes's formula we have

$$
\int_{O}\left\langle\nabla g, \nabla f_{1}\right\rangle=-\int_{O} g \Delta f_{1}
$$

Using the equality $\Delta f_{1} \equiv 0$ and the same arguments as in the proof of Theorem 1 , we arrive at the inequality

$$
\int_{O} \varphi^{2}(m)\left|\nabla f_{1}(m)\right|^{2} \leq 4 \int_{O}\left(f_{1}(m)-\mu_{0}\right)^{2}|\nabla \varphi|^{2} .
$$

Hence we obtain

$$
\int_{F}\left|\nabla f_{1}\right|^{2} \leq 4 \int_{O \backslash(P \cup Q)}\left(f_{1}-\mu_{0}\right)^{2}|\nabla \varphi|^{2} .
$$

By the maximum principle

$$
f_{1}^{2}(m) \leq \mu^{2}(T) \text { for all } m \in O \backslash(P \cup Q),
$$

and from (11) it follows that

$$
\int_{F}\left|\nabla f_{1}\right|^{2} \leq 4\left(\mu(T)-\mu_{0}\right)^{2} \operatorname{cap}(P, Q) .
$$

Using Lemma 2, we have

$$
\int_{F}\left|\nabla f_{1}\right|^{2} \leq\left(\mu(T)-\mu_{0}\right)^{2} \frac{4 I}{T-t^{\prime}},
$$

where $I$ is a constant independent of $T$.

Suppose (10) does not hold. Then from the given inequality it follows that everywhere on $F$ we have $\left|\nabla f_{1}\right|=0$. Since the choice of $F \subset O$ is arbitrary, we conclude that $f_{1}=$ const on $O$. But this contradicts the choice of the point $m_{1} \in O$ at which $f_{1}\left(m_{1}\right)>0$.

\section{$\S 5$}

Theorems 1 and 5 are the geometrical versions of Liouville's theorem and the Phragmén-Lindelöf theorem for harmonic functions on tubular minimal surfaces. The next theorem is an analog of a well-known theorem about removable isolated singularities, and says that a tubular minimal surface with projection $(a,+\infty)$ included between two parallel hyperplanes must be "flattened" at infinity.

THEOREM 6. If a two-dimensional doubly-connected tubular minimal surface with projection $(a,+\infty)$ is situated in the layer $\left|x_{1}\right| \leq 1$, then the oscillation of $x_{1}$ on the set $\Sigma_{e_{0}}(t)$ tends to zero as $t \rightarrow+\infty$.

We carry out the proof of this theorem in parallel with the proof of the analogous assertion for minimal bands for which the $\Sigma_{e_{0}}(t)$ are connected sets.

THEOREM $6^{\prime}$. If a two-dimensional minimal band with projection $(a,+\infty)$ is situated in the layer $\left|x_{1}\right| \leq 1$ and $\left\langle e_{1}, v\right\rangle=0$ everywhere on the part of $\partial M$ not lying in $\Sigma_{e_{0}}(a)$, then the oscillation of $x_{1}$ on the set $\Sigma_{e_{0}}(t)$ tends to zero as $t \rightarrow+\infty$.

The proofs of both assertions follow from a more general assertion presented below, and from the results of [13] concerning the generalized length and area principle. 
LEMMA 3. If $(M, u)$ is a two-dimensional tubular minimal surface with projection $(a,+\infty)$ (or a band, not necessarily two-dimensional), and if the other conditions of Theorem 6 (respectively Theorem $6^{\prime}$ ) are satisfied, then the Dirichlet integral of the function $f_{1}(m)$ is bounded, or

$$
\int_{M(a,+\infty)}\left|\nabla f_{1}\right|^{2}<\infty
$$

For without loss of generality we may assume that $a<b$ are regular values of the function $f=\left\langle u, e_{0}\right\rangle$. We have

$$
\begin{aligned}
\int_{M(a, b)}\left|\nabla f_{1}\right|^{2} & =\int_{\partial M(a, b)} f_{1}\left\langle e_{1}, \nu\right\rangle \\
& =\int_{\Sigma_{e_{0}}(b)} f_{1}\left\langle e_{1}, \nu\right\rangle-\int_{\Sigma_{e_{0}}\langle a)} f_{1}\left\langle e_{1}, \nu\right\rangle+\int_{\partial M \cap M(a, b)} f_{1}\left\langle e_{1}, \nu\right\rangle .
\end{aligned}
$$

The last integral is equal to zero by virtue of the assumptions in the conditions of Theorems 6 and $6^{\prime}$, and so

$$
\int_{M(a, b)}\left|\nabla f_{1}\right|^{2}=\int_{\Sigma_{e_{0}}(b)} f_{1}\left\langle e_{1}, \nu\right\rangle-\int_{\Sigma_{e_{0}}(a)} f_{1}\left\langle e_{1}, \nu\right\rangle .
$$

From the Kronrod-Federer formula ([17], p. 103), using Cauchy's inequality, we have

$$
\int_{M(a, b)}\left|\nabla f_{1}\right|^{2}=\int_{a}^{b} d t \int_{\Sigma_{\varepsilon_{0}}(t)} \frac{\left|\nabla f_{1}\right|^{2}}{|\nabla f|} \geq \int_{a}^{b} d t\left(\int_{\Sigma_{e_{0}}(t)}\left|\nabla f_{1}\right|\right)^{2}\left(\int_{\Sigma_{e_{0}}(t)}|\nabla f|\right)^{-1} .
$$

But we showed earlier that for any $\alpha$ and $\beta$ such that $a \leq \alpha<\beta$ we have

$$
\int_{M(\alpha, \beta)}|\nabla f|^{2}=I(\beta-\alpha) \text {. }
$$

so we conclude that

$$
\int_{M(a, b)}\left|\nabla f_{1}\right|^{2}=\frac{1}{I} \int_{a}^{b} \varphi^{2}(t) d t
$$

where

$$
\varphi(t)=\int_{\Sigma_{e_{0}}(t)}\left|\nabla f_{1}\right|
$$

We now prove that the integral on the right-hand side of (13) is bounded independently of $b$. For this we use the fact that $\left|f_{1}(m)\right| \leq 1$ on $M$, and so by (12)

$$
\int_{M(a, b)}\left|\nabla f_{1}\right|^{2} \leq-\int_{\Sigma_{e_{0}}(a)} f_{1}\left\langle e_{1}^{T}, \nu\right\rangle+\int_{\Sigma_{e_{0}}(b)}\left|e_{1}^{T}\right|=-\int_{\Sigma_{e_{0}}(a)} f_{1}\left\langle e_{1}^{T}, \nu\right\rangle+\varphi(b) .
$$

Using (13), we obtain

$$
\int_{a}^{b} \varphi^{2}(t) d t \leq I \varphi(t)-I \int_{\Sigma_{e_{0}}(a)} f_{1}\left\langle e_{1}^{T}, \nu\right\rangle
$$

If here we assume that for some $t_{0}>a$

$$
\Phi\left(t_{0}\right)>-I \int_{\Sigma_{e_{0}}(a)}\left\langle e_{1}, \nu\right\rangle f_{1}=c, \quad \Phi(t)=\int_{a}^{t} \varphi^{2}(\tau) d \tau,
$$


then in an obvious way for $t>t_{0}$ we obtain

$$
I \varphi(t)>\Phi(t)-c>0
$$

and so, using the fact that $\varphi^{2}(t)=\Phi^{\prime}(t)$,

$$
\frac{\Phi^{\prime}(t)}{(\Phi(t)-c)^{2}}>I \quad \text { when } t>t_{0}
$$

Integrating this from $t_{0}$ to $t>t_{0}$, we obtain

$$
\left(t-t_{0}\right) I<\frac{1}{\Phi\left(t_{0}\right)-c}-\frac{1}{\Phi(t)-c}<\frac{1}{\Phi\left(t_{0}\right)-c},
$$

which contradicts the fact that $t$ is unbounded (because $I \neq 0$ by (13)). Consequently,

$$
\Phi(t)=\int_{a}^{t} \varphi^{2}(\tau) d \tau \leq-\int_{\Sigma_{e_{0}}(a)} f_{1}\left\langle e_{1}, \nu\right\rangle .
$$

Next, from the fact that the integral $\int_{a}^{t} \varphi^{2}(\tau) d \tau$ converges, we conclude that there is a sequence $t_{k} \nearrow \infty$ such that $\varphi\left(t_{k}\right) \rightarrow 0$ and therefore, passing to the limit as $k \rightarrow \infty$ in the inequality

$$
\int_{M\left(a, t_{k}\right)}\left|\nabla f_{1}\right|^{2} \leq-\int_{\Sigma_{e_{0}}(a)} f_{1}\left\langle e_{1}, \nu\right\rangle+\varphi\left(t_{k}\right)
$$

we see that the integral

$$
\int_{M(a,+\infty)}\left|\nabla f_{1}\right|^{2} \leq-\int_{\Sigma_{e_{0}}(a)} f_{1}\left\langle e_{1}, \nu\right\rangle
$$

is bounded, as required.

Now for the proof of Theorems 6 and $6^{\prime}$ we use a result of [13], p. 256. Namely, it follows from Lemma 3 that

$$
\inf _{\xi_{1}<t<\xi_{2}} \underset{m \in \Sigma_{e_{0}}(t)}{\operatorname{osc}} 2\left(f_{1}(m)\right) \leq \operatorname{cap}\left(P_{\xi_{1}}, Q_{\xi_{2}}\right) \int_{M\left(\xi_{1}, \xi_{2}\right)}\left|\nabla f_{1}\right|^{2}
$$

for any $a<\xi_{1}<\xi_{2}<\infty$. Hence there is a sequence $t_{k} \nearrow \infty$ such that

$$
\underset{m \in \Sigma_{e_{0}}(t)}{\operatorname{osc}}\left(f_{1}(m)\right) \rightarrow 0 \text {. }
$$

Since $\left|f_{1}(m)\right|$ is bounded on $M(a,+\infty)$ and $\left\langle e_{1}, \nu\right\rangle=0$ on the part of $\partial M$ not lying in $\Sigma_{e_{0}}(a)$, using the maximum principle for harmonic functions (and if necessary going over to the subsequence $t_{k}$ ) we conclude that

$$
\lim _{k \rightarrow \infty} \sup _{m \in \Sigma_{e_{0}}\left(t_{k}\right)} f_{1}(m)=\lim _{k \rightarrow \infty} \inf _{m \in \Sigma_{e_{0}}\left(t_{k}\right)} f_{1}(m)=c .
$$

But

$$
\sup _{m \in M\left(t_{k}, t_{k+1}\right)} f_{1}(m)=\max \left[\sup _{m \in \Sigma_{e_{0}}\left(t_{k}\right)} f_{1}(m) ; \sup _{m \in \Sigma_{e_{0}}\left(t_{k+1}\right)} f_{1}(m)\right],
$$

and the same for the minimum. Consequently,

$$
\lim _{k \rightarrow \infty} \underset{M\left(t_{k}, t_{k+1}\right)}{\text { osc }} f_{1} \leq \lim _{k \rightarrow \infty} \underset{\Sigma_{e_{0}}\left(t_{k}\right)}{\text { osc }} f_{1}=0
$$


from which it follows that

$$
\lim _{k \rightarrow \infty} \underset{M\left(t_{k}, \infty\right)}{\text { osc }} f_{1}=0
$$

which finally proves both theorems.

It is obvious from the proof of Theorem 2 that in the case $p \geq 3$ on any $p$ dimensional minimal surface in $\mathbf{R}^{n}$ there exist a priori superharmonic functions, bounded below, which leads to a contradiction with the fact that the type is parabolic. Hence the geometrical structure of a tubular minimal surface when $p \geq 3$ differs in principle (in a well-defined sense) from the two-dimensional case.

First of all we prove the following auxiliary assertion. Choosing a suitable coordinate system, we may assume, without loss of generality, that the point $u=0$ does not lie in the image $u(M)$.

Lemma 4. Let $(M, u)$ be a p-dimensional minimal surface in $\mathbf{R}^{n}$ and suppose that $p \geq 3$. Then the function $g(m)=|u(m)|^{2-p}$ is superharmonic.

We put $\rho=\rho(m)=|u(m)|$ and for a fixed basis of orthonormal vectors $e_{1}, \ldots, e_{n}$ in $\mathbf{R}^{n}$ we denote by $u_{i}=\left\langle u, e_{i}\right\rangle$ a set of coordinate functions. Then for any tangent vector $X \in T_{m}(M)$ we have

$$
\nabla_{X} u_{i}=\left\langle\bar{\nabla}_{X} u, e_{i}\right\rangle=\left\langle X, e_{i}^{T}\right\rangle,
$$

from which it is obvious that $\nabla u_{i}=e_{i}^{T}$. Next, from the fact that the surface $(M, u)$ is minimal it follows that $\Delta u_{i} \equiv 0$, and thus

$$
\Delta \rho^{2}(m)=\Delta \sum_{i=1}^{n} u_{i}^{2}(m)=2 \sum_{i=1}^{n}\left(u_{i} \Delta u_{i}+\left|\nabla u_{i}\right|^{2}\right)=2 \sum_{i=1}^{n}\left|e_{i}^{T}\right|^{2}=2 p .
$$

On the other hand, we see that

$$
\Delta \rho^{2}(m)=2 \rho(m) \Delta \rho(m)+2|\nabla \rho(m)|^{2},
$$

and so from what we found above we have $\Delta \rho=\left(p-|\nabla \rho|^{2}\right) / \rho$. Let us find the value of the Laplacian of the function we need:

$$
\begin{aligned}
\Delta\left(\rho^{2-p}\right) & =\operatorname{div}\left(-\frac{p-2}{\rho^{p-1}} \nabla \rho\right)=-\frac{p-2}{\rho^{p-1}} \Delta \rho+\frac{(p-2)(p-1)}{\rho^{p}}|\nabla \rho|^{2} \\
& =-\frac{p-2}{\rho^{p}}\left(p-p|\nabla \rho|^{2}\right) \leq 0,
\end{aligned}
$$

in view of the fact that $|\nabla \rho|=\left|u^{T}\right| / \rho \leq|u| / \rho=1$ everywhere on $M$. It is obvious that $g(m)=\rho^{2-p}(m)>0$; that is, $g(m)$ is a superharmonic function bounded below, as required.

For the proof of Theorem 2 we now observe that if $g(m)$ is superharmonic, then the function

$$
h(t)=\inf _{m \in \Sigma_{e_{0}}(t)} g(m)=\left[\max _{m \in \Sigma_{e_{0}}(t)} \rho(m)\right]^{2-p}
$$

(defined for all $t \in(a, b)$, where $(a, b)$ is the interval of projection of the tubular minimal surface) is convex upwards. For suppose that $a<\alpha<\beta<b$; then the 
function

$$
g_{1}(m)=g(m)-\left(h(\alpha)+\frac{h(\beta)-h(\alpha)}{\beta-\alpha}(f(m)-\alpha)\right),
$$

where $f(m)=\left\langle u, e_{0}\right\rangle$, is also superharmonic (as a linear combination of a superharmonic and a harmonic function with constant coefficients), and

$$
\begin{aligned}
& \min _{m \in \Sigma_{e_{0}}(\alpha)} g_{1}(m)=\min _{m \in \Sigma_{e_{0}}(\alpha)} g(m)-h(\alpha)=0, \\
& \min _{m \in \Sigma_{e_{0}}(\beta)} g_{1}(m)=\min _{m \in \Sigma_{e_{0}}(\beta)} g(m)-h(\beta)=0 .
\end{aligned}
$$

Applying the usual maximum principle for the superharmonic function $g_{1}(m)$, for all $m \in M(\alpha, \beta)$ we have

$$
g_{1}(m) \geq \min _{\tau \in \Sigma_{e_{0}}(\alpha) \cup \Sigma_{e_{0}}(\beta)} g_{1}(\tau)=0,
$$

that is,

$$
h(t) \geq h(\alpha)+\frac{h(\beta)-h(\alpha)}{\beta-\alpha}(t-\alpha),
$$

which by definition implies that $h(t)$ is convex upwards.

We now suppose that one of the numbers $a$ and $b$ is equal to $\pm \infty$. Without loss of generality we may assume that $b=+\infty$. In this case there is a limit

$$
\lim _{t \rightarrow+\infty} h(t)=\lim _{t \rightarrow+\infty}\left(\sup _{m \in \Sigma_{e_{0}}(t)} \rho(m)\right)^{2-p}=0,
$$

which by a well-known property of the convex upwards function $h(t)$ contradicts the fact that it is nonnegative. Consequently, the two numbers $a$ and $b$ are finite, and the tubular minimal surface is in the layer between the hyperplanes $\Pi_{a}$ and $\Pi_{b}$, which completes the proof of Theorem 2.

Let us proceed to the proof of Theorem 3. As in the previous cases, the proof relies on the existence of a superharmonic function with the necessary properties.

Suppose there is a two-dimensional plane $V \in G_{n}^{2}$ such that $\sup _{m^{*} \in M^{*}} \theta(\gamma, V)=$ $\theta_{0}<\pi / 2$. We choose an arbitrary unit vector $e \in V$ and fix a parallel (in $\mathbf{R}^{n}$ ) vector field $e=e(m)$ that can be identified at each point $m \in M$ with the vector $e$. Consider the function $\varphi(m)=\ln \left|e^{T}\right|=\ln \left|e^{T_{m}(M)}\right|$. We observe that under our assumptions $\left|e^{T_{m}(M)}\right| \geq \cos \theta\left(T_{m}(M), V\right) \geq \cos \theta_{0}>0$, so $\varphi=\varphi(m)$ is defined on the whole of $M$ and is bounded below. For any $X \in T_{m}(M)$ we have

$$
\begin{aligned}
\nabla_{X} \varphi(m) & =\frac{1}{\left|e^{T}\right|} \nabla_{X}\left|e^{T}\right|=\frac{\left\langle e^{T}, \bar{\nabla}_{X} e^{T}\right\rangle}{\left|e^{T}\right|^{2}}=\frac{1}{\left|e^{T}\right|}=\left\langle\tau,-\bar{\nabla}_{X} e^{N}\right\rangle \\
& =\frac{1}{\left|e^{T}\right|}\left\langle\tau, A^{e^{N}} X\right\rangle=\left\langle X, \frac{1}{\left|e^{T}\right|} A^{e^{N}} \tau\right\rangle,
\end{aligned}
$$

where $\tau=e^{T} /\left|e^{T}\right|$, and so

$$
\Delta \varphi=\frac{1}{\left|e^{T}\right|} A^{e^{N}}(\tau)
$$


We now use Lemma 1. For this we choose an arbitrary orthonormal basis $E_{1}, E_{2}$ in the tangent space $T_{m}(M)$ and from the definition of divergence we obtain

$$
\Delta \varphi=\frac{1}{\left|e^{T}\right|} \operatorname{div} A^{e^{N}} \tau+\left(-\frac{1}{\left|e^{T}\right|^{2}}\right)\left\langle\nabla\left|e^{T}\right|, A^{e^{N}} \tau\right\rangle .
$$

It is easy to find the second term by using (14), since it is not difficult to see that $\nabla\left|e^{T}\right|=\left|e^{T}\right| \nabla \varphi=A^{e^{N}}(\tau)$, and consequently

$$
\Delta \varphi=\frac{1}{\left|e^{T}\right|} \operatorname{div} A^{e^{N}} \tau-\frac{1}{\left|e^{T}\right|^{2}}\left|A^{e^{N}} \tau\right|^{2} .
$$

By Lemma 1 the first term can be calculated from the formula

$$
\operatorname{div} A^{e^{N}} \tau=\sum_{i=1}^{2}\left(\left\langle A^{e^{N}} E_{i}, \nabla_{E_{i}} \tau\right\rangle+\left\langle B\left(\tau, E_{i}\right), \nabla_{E_{i}} e^{N}\right\rangle\right)
$$

We immediately find that

$$
\nabla_{E_{i}} e^{N}=\left(\bar{\nabla}_{E_{i}}\left(e-e^{T}\right)\right)^{N}=-B\left(e^{T}, E_{i}\right)=-\left|e^{T}\right| B\left(\tau, E_{i}\right)
$$

and also

$$
\begin{aligned}
\nabla_{E_{i}} \tau & =\nabla_{E_{i}}\left(\frac{e^{T}}{\left|e^{T}\right|}\right)=\frac{\left|e^{T}\right| \nabla_{E_{i}} e^{T}-e^{T} \nabla_{E_{i}}\left|e^{T}\right|}{\left|e^{T}\right|^{2}}=\frac{\left|e^{T}\right| A^{e^{N}} E_{i}-e^{T}\left\langle A^{e^{N}} \tau, E_{i}\right\rangle}{\left|e^{T}\right|^{2}} \\
& =\frac{1}{\left|e^{T}\right|}\left(A^{e^{N}} E_{i}-\tau\left\langle A^{e^{N}} E_{i}, \tau\right\rangle\right) .
\end{aligned}
$$

Substituting what we have found in (16), we obtain

$$
\begin{aligned}
\operatorname{div} A^{e^{N}} \tau & =\sum_{i=1}^{2}\left(\frac{\left|A^{e^{N}} E_{i}\right|^{2}-\left\langle A^{e^{N}} E_{i}, \tau\right\rangle^{2}}{\left|e^{T}\right|}-\left|e^{T}\right|\left|B\left(\tau, E_{i}\right)\right|^{2}\right) \\
& =\frac{\sum_{i=1}^{2}\left|A^{e^{N}} E_{i}\right|^{2}-\left|A^{e^{N}} \tau\right|^{2}}{\left|e^{T}\right|}-\frac{1}{2}\|B\|^{2}\left|e^{T}\right|,
\end{aligned}
$$

where $\|B\|^{2}=\sum_{i, j=1}^{2}\left|B\left(E_{i}, E_{j}\right)\right|^{2}$ is the length of the second quadratic form.

We immediately verify a special property of the map $A^{\xi}$ of a two-dimensional minimal surface, namely,

$$
\left|A^{\xi} E_{1}\right|^{2}=\left|A^{\xi} E_{2}\right|^{2}=\left|A^{\xi} X\right|^{2}, \quad \forall \xi \in N_{m}(M), X \in T_{m}(M),
$$

from which, using (15) and (17), we obtain

$$
\Delta \varphi=\frac{\left|A^{e^{N}} \tau\right|^{2}}{\left|e^{T}\right|^{2}}-\frac{1}{2}\|B\|^{2}-\frac{\left|A^{e^{N}} \tau\right|^{2}}{\left|e^{T}\right|^{2}} .
$$

From (18) it is obvious that $\varphi(m)$ is a superharmonic function, and as we said above $\varphi(m) \geq \ln \left(\cos \theta_{0}\right)>-\infty$. Using arguments analogous to those in the proof of Theorem 1, and the fact that the surface is tubular in the large, we conclude that $\left|e^{T}\right| \equiv$ const $\neq 0$ on $M$, and finally $\Delta \varphi \equiv 0 \equiv-\|B\|^{2} / 2$; that is, $\|B\| \equiv 0$. From the last equality it follows that the surface $(M, u)$ can only be a plane, which contradicts the fact that the surface $(M, u)$ is tubular. Consequently, the assumption made at 
the beginning of the proof about the existence of the plane $V$ is not true, and so for any $V \in G_{m}^{2}$ we have $\sup _{\gamma \in M^{*}} \theta(\gamma, V)=\pi / 2$.

$$
\S 8
$$

We observe that the methods we have used above can be used to study the structure of the Gaussian image of a minimal surface of tubular type close to infinity, in terms of the ends of the surface $(M, u)$.

Consider a tubular surface $(M, u)$ in $\mathbf{R}^{n}$ with projection $(a,+\infty)$. An arbitrary noncompact doubly-connected set $D \subset M$ with compact boundary $\partial D$ is called an end of the surface $(M, u)$. We introduce the following definition of the limit set of an end of the surface $D$ : we put

$$
D^{\prime}=\bigcap_{t>a} \overline{D^{*}(t,+\infty)}
$$

where $D^{*}(t, \infty)$ denotes the Gaussian image of part of the end $D \cap M(t, \infty)$. Clearly $D^{\prime}$ is a nonempty closed set in $G_{n}^{2}$.

THEOREM 7. Let $(M, u)$ be a two-dimensional tubular minimal surface in $\mathbf{R}^{n}$ with projection $(a,+\infty)$, and $D$ one of the ends of $(M, u)$. Then either

( $\alpha) D^{\prime}$ intersects any equator of $G_{n}^{2}$, or

( $\beta$ ) $D^{\prime}$ consists of a unique point $\gamma \in G_{n}^{2}$, and in this case $\theta\left(e_{0}, \gamma\right)=\pi / 2$.

For the proof we suppose that $(\alpha)$ does not hold, and without loss of generality we assume that there exist $V \in G_{n}^{2}$ and $K_{V} \cap D^{\prime}=\varnothing$ such that $D^{\prime}$ lies (since it is closed) in one half of $G_{n}^{2} \backslash K_{V}$ together with $V$. Hence it follows immediately that $\max _{\gamma \in D^{\prime}} \theta(\gamma, V)=\theta_{0}<\pi / 2$. Let $\varepsilon>0$ be chosen so that $\varepsilon<\pi / 2-\theta_{0}$. Then by the definition of $D^{\prime}$ we can choose $T>a$ large enough so that for all $\gamma \in$ $\overline{D^{*}(T,+\infty)}$ we have $\theta(\gamma, V)<\pi / 2-\varepsilon$. We observe that from the last inequality and transversality properties it follows that the chosen set $D(T, \infty)$ is homeomorphic to an annulus.

Consider the set of unit vectors of $\mathbf{R}^{n}$

$$
O=\left\{e \in S^{n-1}:\left|e^{V}\right|>\cos \varepsilon\right\},
$$

where $S^{n-1} \subset \mathbf{R}^{n}$ is the sphere of unit radius with center at the origin. Clearly, $O$ is an open subset of $S^{n-1}$.

For any $\gamma \in G_{n}^{2}$ and $e \in \mathbf{R}^{n}$ we have

$$
\theta(e, \gamma) \leq \theta(e, V)+\theta(V, \gamma),
$$

where we keep the same notation for the angle between a vector and a plane. Consequently, for any $e \in O$ and $\gamma \in \overline{D^{*}(T,+\infty)}$ we have $\theta(e, \gamma) \leq \varepsilon+\theta_{0}<\pi / 2$, and therefore $\left|e^{\gamma}\right|>\cos \left(\varepsilon+\theta_{0}\right)>0$.

We showed above that for any $e_{1}, e_{2} \in \mathbf{R}^{n}$ we have

$$
\Delta \ln \left|e_{i}^{T_{m}(M)}\right|=-\frac{1}{2}\|B\|^{2},
$$

from which it is obvious that the function $\varphi(m)=\ln \left(\left|e_{1}^{T_{m}}\right| /\left|e_{2}^{T_{m}}\right|\right)$ is harmonic in the domain of definition. But, by the remarks we made above, for any $e_{1}, e_{2} \in O$ with $e_{1} \neq e_{2}$ we have

$$
|\varphi(m)|=\left|\ln \frac{\left|e_{1}^{T_{m}}\right|}{\left|e_{2}^{T_{m}}\right|}\right| \leq-\ln \cos \left(\theta_{0}+\varepsilon\right) ;
$$


that is, $\varphi(m)$ is bounded on $D(T,+\infty)$. We show that there is a limit

$$
\lim _{t \rightarrow \infty} \underset{m \in \Sigma_{e_{0}}(t)}{\operatorname{osc}} \varphi(m)=0
$$

or equivalently that the function $\varphi(m)$ has a limit at infinity.

For this we observe that up to a constant multiplier $\varphi(m)$ satisfies the conditions of Theorem 6, and also that in the proof of this theorem we have essentially used the fact that $f_{1}(m)$ is harmonic and bounded. Consequently, replacing $f_{1}(m)$ by $\varphi(m)$ everywhere, we obtain the required relation (19). In terms of the limit set $D^{\prime}$ this simply means that for each pair of fixed vectors $e_{1}, e_{2} \in O$ the quantity $\left|e_{1}^{\gamma}\right| /\left|e_{2}^{y}\right|$ does not depend on $\gamma \in G_{n}^{2}$. We show that $D^{\prime}$ consists of a single point. To this end we assume the contrary; that is, there are at least two planes $\gamma, \xi$ such that $\gamma \neq \xi$. Then for any $e_{1}, e_{2} \in O$ we have

$$
\left|e_{1}^{\gamma}\right| /\left|e_{2}^{\gamma}\right|=\left|e_{1}^{\xi}\right| /\left|e_{2}^{\xi}\right| .
$$

Consider a map $h: \mathbf{R}^{n} \rightarrow \mathbf{R}^{1}$ of the form

$$
h(x)=\left|x^{\gamma}\right| /\left|x^{\xi}\right|, \quad x^{\xi} \neq 0,
$$

then the restriction $\left.h\right|_{O}(x)$ is identically constant, say $c$. Since $h(x)$ is homogeneous, we conclude that $h(x) \equiv c$ on the open cone $O^{\prime}=\left\{y \in \mathbf{R}^{n}: y /|y| \in O, y \neq\right.$ $0\}$. However, $h(x)$ is an analytic function in its domain, and so by the uniqueness theorem for analytic functions $h(x) \equiv$ const $=c$.

By our assumption $\xi \neq \gamma$, so for their orthogonal complements we have $\gamma^{\perp} \backslash \xi^{\perp} \neq$ $\varnothing$. Choosing $e \in \gamma^{\perp} \backslash \xi^{\perp}$, we thus obtain $c=\left|e^{\gamma}\right| /\left|e^{\xi}\right| \neq 0$, which is impossible, since we obviously have $h(x) \neq 0$ for $x \in \gamma$.

In the case when $D^{\prime}$ consists of a single point, we can assert that $D^{\prime}=\gamma \in G_{n}^{2}$ and $\left|e_{0}^{\gamma}\right|=0$.

For suppose the contrary; that is, $\left|e^{\gamma}\right|=\cos \alpha \neq 0$, where $0 \leq \alpha<\pi / 2$. Then by the definition of $D^{\prime}$ there is a sufficiently large $A>a$ such that

(a) $A$ is a regular value of the function $f(m)=\left\langle u(m), e_{0}\right\rangle$, and

(b) for any $m \in D(A, \infty)$ we have $\theta\left(\gamma, T_{m}\right)<\varepsilon<\pi / 2-\alpha$.

By virtue of (a) the set $D \cap \Sigma_{e_{0}}(A)$ is a one-dimensional compact manifold in $M$; that is, it splits into the union of finitely many smooth closed Jordan arcs. From them we choose an arbitrary arc $\Gamma_{\varepsilon}$ and denote by $\tau=\tau(m)$ its tangent vector at the point $m \in \Gamma_{\varepsilon}$. By our assumption, $e_{0}^{\gamma} \neq 0$, so we can choose a unit vector $v \in \gamma$ such that $\left\langle e_{0}^{\gamma}, v\right\rangle=0$.

We observe that by the definition of $\Gamma_{\varepsilon} \subset D \cap \Sigma_{e_{0}}(A)=\{m \in D: f(m)=A\}$ the vector field $e^{T_{m}(M)}$ is normal to $\Gamma_{\varepsilon}$ in $M$, and so the vector fields $\tau(m)$ and $e^{T_{m}}$ form an orthogonal basis in $T_{m}(M)$. We show that we can choose $\varepsilon>0$ so that for any $m \in \Gamma_{\varepsilon}$ the quantity $\langle v, \tau(m)\rangle$ preserves its sign; that is, $\langle v, \tau(m)\rangle \neq 0$.

For otherwise, for any $\varepsilon>0$ there would be an $m \in \Gamma_{\varepsilon}$ for which $V^{T_{m}}=e_{0}^{T_{m}} \mu$, where $\mu$ is a nonzero real number (since $\left|v^{T_{m}}\right| \geq \cos \theta\left(T_{m}, \gamma\right)>0$ ), depending on $m$. From the last equality it is obvious that $\left(v-\mu e_{0}\right)^{T_{m}}=0$; that is, the vector $w=v-\mu e_{0}$ lies in $N_{m}(M)$. We have

$$
\langle w, v\rangle=\langle v, v\rangle-\mu\left\langle e_{0}, v\right\rangle=1-\mu\left\langle e_{0}^{\gamma}, v\right\rangle=1
$$


since $\left\langle e_{0}^{\gamma}, v\right\rangle=0$ by the choice of $v \in \gamma$. But then, since $w \in N_{m}$,

$$
1 \equiv|\langle w, v\rangle|=\left|\left\langle w^{N}, v^{N}\right\rangle\right| \leq\left|w^{N}\right|\left|v^{N}\right| \text {. }
$$

We observe that $\left|w^{N}\right|=|w|=\left|v-\mu e_{0}\right| \leq 1+|\mu|$. On the other hand,

$$
\left\langle w, e_{0}^{T}\right\rangle=\left\langle v, e_{0}^{T}\right\rangle-\left|e_{0}^{T}\right| 2 \mu=0 \mid \Rightarrow \mu=\frac{\left\langle v, e_{0}^{T}\right\rangle}{\left|e_{0}^{T}\right|^{2}} .
$$

Hence we get the estimate

$$
|\mu|=\frac{\left|\left\langle v, e_{0}^{T}\right\rangle\right|}{\left|e_{0}^{T}\right|^{2}} \leq \frac{|v|\left|e_{0}^{T}\right|}{\left|e_{0}^{T}\right|^{2}}=\frac{1}{\left|e_{0}^{T}\right|} .
$$

By virtue of our choice

$$
\theta\left(e_{0}, T_{m}\right) \leq \theta\left(e_{0}, \gamma\right)+\theta\left(\gamma, T_{m}\right) \leq \alpha+\varepsilon<\pi / 2
$$

and thus

$$
\left|e_{0}^{T_{m}}\right|=\cos \theta\left(e_{0}, T_{m}\right) \geq \cos (\alpha+\varepsilon)>0
$$

Inequality (21) takes the form $|\mu|<1 / \cos (\alpha+\varepsilon)$, and in conjunction with (20) it gives the relation

$$
1 \leq\left|v^{N}\right|(1+|\mu|) \leq\left(1-\left|v^{T}\right|^{2}\right)^{1 / 2}\left(1+\frac{1}{\cos (\alpha+\varepsilon)}\right) \leq \sin \varepsilon \frac{1+\cos (\alpha+\varepsilon)}{\cos (\alpha+\varepsilon)}
$$

From (22) it is obvious that for a suitable choice of $\varepsilon>0$ we arrive at a contradiction, since the right-hand side of this inequality can be made arbitrarily small as $\varepsilon \rightarrow 0$. We choose $\varepsilon>0$ so that

$$
\sin \varepsilon \frac{1+\cos (\alpha+\varepsilon)}{\cos (\alpha+\varepsilon)}<1
$$

Then for any $m \in \Gamma_{\varepsilon}$ we have $\langle v, \tau(m)\rangle \neq 0$, and so $\langle v, \tau(m)\rangle$ preserves its sign on $\Gamma_{\varepsilon}$, say positive. Consider the coordinate function $g(m)=\langle v, u(m)\rangle$ with direction vector $v$ and gradient $\nabla g=v^{T_{m}}$. Then for $m \in \Gamma_{\varepsilon}$ we have

$$
\langle\nabla g, \tau(m)\rangle=\left\langle v^{T_{m}}, \tau(m)\right\rangle=\langle v, \tau(m)\rangle>0 .
$$

Integrating this over $\Gamma_{\varepsilon}$, we have

$$
0<\int_{m \in \Gamma_{\varepsilon}}\langle\nabla g(m), \tau(m)\rangle=\int_{\Gamma_{\varepsilon}} d g(m)=0 .
$$

This contradiction proves the theorem.

Volgograd State University

Received 23/MAY/88 and 25/AUG/88

\section{BIBLIOGRAPHY}

1. Shoshichi Kobayashi and Katsumi Nomizu, Foundations of differential geometry. Vol. 2, Interscience, 1969.

2. Johannes C. C. Nitsche, A uniqueness theorem of Bernstein's type for minimal surfaces in cylindrical coordinates, J. Math. and Mech. 6 (1957), 859-864.

3. A. D. Vedenyapin and V. M. Miklyukov, Extrinsic dimensions of tubular minimal hypersurfaces, Mat. Sb. 131(173) (1986), 240-250; English transl. in Math. USSR Sb. 59 (1988).

4. V. M. Miklyukov, On some properties of tubular minimal surfaces in $\mathbf{R}^{n}$, Dokl. Akad. Nauk SSSR 247 (1979), 549-552; English transl. in Soviet Math. Dokl. 20 (1979).

5. __. On a new approach to Bernstein's theorem and similar questions for equations of minimal surface type, Mat. Sb. 108(150) (1979), 268-289; English transl. in Math. USSR Sb. 36 (1980). 
6. A. A. Grigor'yan, On the existence of positive fundamental solutions of the Laplace equation on Riemannian manifolds, Mat. Sb. 128(170) (1985), 354-363; English transl. in Math. USSR Sb. 56 (1987).

7. James Simons, Minimal varieties in Riemannian manifolds, Ann. of Math. (2) 88 (1968), 62-105.

8. V. M. Miklyukov and V. G. Tkachev, On the structure in the large of externally complete minimal surfaces in $\mathbf{R}^{3}$, Izv. Vyssh. Uchebn. Zaved. Mat. 1987, no. 7 (302), 30-36; English transl. in Soviet Math. (Iz. VUZ) 31 (1987).

9. V. G. Tkachev, Tests for parabolicity of conformal type of two-dimensional minimal surfaces in $\mathbf{R}^{n}$, All-Union Conf. Geometry "in the Large", Abstracts of Reports, Inst. Mat. Sibirsk. Otdel. Akad. Nauk SSSR, Novosibirsk, 1987, p. 119. (Russian)

10. B. M. Barbashov and V. V. Nesterenko, A model of a relativistic string in the physics of hadrons, Energoatomizdat, Moscow, 1987. (Russian)

11. _- Superstrings - a new approach to a unified theory of fundamental interactions, Uspekhi Fiz. Nauk 150 (1986), 489-524; English transl. in Soviet Phys. Uspekhi 29 (1986), no. 12.

12. R. Courant, Dirichlet's principle, conformal mapping, and minimal surfaces, Interscience, 1950.

13. G. D. Suvorov, The generalized "length and area principle" in the theory of mappings, "Naukova Dumka", Kiev, 1985. (Russian)

14. A. T. Fomenko, On the rate of growth and least volumes of globally minimal surfaces in cobordisms, Trudy Sem. Vektor. Tenzor. Anal. No. 21 (1983), pp. 3-12. (Russian)

15. - The algebraic structure of certain classes of completely integrable Hamiltonian systems on Lie algebras, Geometrical Theory of Functions and Topology (N. P. Korneichuk, editor), Inst. Mat. Akad. Nauk Ukrain. SSR, Kiev, 1981, pp. 85-126. (Russian) *

16. _ _ Variational methods in topology, "Nauka", Moscow, 1982. (Russian)

17. Yu. D. Burago and V. A. Zalgaller, Geometric inequalities, "Nauka", Leningrad, 1980. (Russian)

18. Atsushi Kasue, Gap theorems for minimal submanifolds of Euclidean space, J. Math. Soc. Japan. 38 (1986), 473-492.

19. Richard M. Schoen, Uniqueness, symmetry, and embeddedness of minimal surfaces, J. Differential Geometry 18 (1983), 791-809.

Translated by E. J. F. PRIMROSE

* Editor's note. According to MR 84c:58034, "This article is essentially a preprint of the author's later paper", Mat. Sb. 115(157) (1981), 263-280; English transl. in Math. USSR Sb. 43 (1982). 